\title{
Improving the photometric precision of IRAC Channel 1
}

Kenneth J. Mighell, William Glaccum, William Hoffmann

Kenneth J. Mighell, William Glaccum, William Hoffmann, "Improving the photometric precision of IRAC Channel 1," Proc. SPIE 7010, Space Telescopes and Instrumentation 2008: Optical, Infrared, and Millimeter, 70102W (12 July 2008); doi: 10.1117/12.789801

EDIE Event: SPIE Astronomical Telescopes + Instrumentation, 2008, Marseille, France 


\title{
Improving the photometric precision of IRAC Channel 1
}

\author{
Kenneth J. Mighell ${ }^{a}$, William Glaccum ${ }^{b}$, and William Hoffmann ${ }^{c}$ \\ ${ }^{a}$ National Optical Astronomy Observatory, 950 N. Cherry Ave., Tucson, AZ 85719, U.S.A.; \\ ${ }^{b}$ Spitzer Science Center, California Institute of Technology, Mail Code 314-6, Pasadena, CA \\ 91125, U.S.A.; \\ ${ }^{c}$ Steward Observatory, University of Arizona, 933 N Cherry Ave., Tucson AZ 85721, U.S.A.
}

\begin{abstract}
Planning is underway for a possible post-cryogenic mission with the Spitzer Space Telescope. Only Channels 1 and $2(3.6$ and $4.5 \mu \mathrm{m})$ of the Infrared Array Camera (IRAC) will be operational; they will have unmatched sensitivity from 3 to 5 microns until the James Webb Space Telescope is launched. At SPIE Orlando, Mighell described his NASA-funded MATPHOT algorithm for precision stellar photometry and astrometry and presented MATPHOT-based simulations that suggested Channel 1 stellar photometry may be significantly improved by modeling the nonuniform RQE within each pixel, which, when not taken into account in aperture photometry, causes the derived flux to vary according to where the centroid falls within a single pixel (the pixel-phase effect). We analyze archival observations of calibration stars and compare the precision of stellar aperture photometry, with the recommended 1-dimensional and a new 2-dimensional pixel-phase aperture-flux correction, and MATPHOT-based PSF-fitting photometry which accounts for the observed loss of stellar flux due to the nonuniform intrapixel quantum efficiency. We show how the precision of aperture photometry of bright isolated stars corrected with the new 2-dimensional aperture-flux correction function can yield photometry that is almost as precise as that produced by PSF-fitting procedures. This timely research effort is intended to enhance the science return not only of observations already in Spitzer data archive but also those that would be made during the Spitzer Warm Mission.
\end{abstract}

Keywords: stellar photometry, astrometry, infrared detectors, Spitzer Space Telescope, IRAC, MATPHOT

\section{INTRODUCTION}

A detector can be considered to be effectively lossy if a pixel, the smallest optically sensitive unit of the detector, internally exhibits a non-uniform response function that has a quantum efficiency variation with an rms dispersion exceeding an arbitrary level of 1\%. By this user-centric definition, the detectors in Channel 1 (Ch1) of the Infrared Array Camera (IRAC) instrument ${ }^{1}$ onboard the Spitzer Space Telescope are lossy ${ }^{2,3}$. Near-infrared astronomical cameras based on lossy detectors can have significant systematic errors in the measurement of total stellar flux and position - if stellar images are undersampled as they are in IRAC Ch1. Precision photometric and astrometric analysis of image data from cameras with undersampled lossy detectors is consequently frequently problematical.

This article describes how the precision of stellar photometry from IRAC Ch1 can be significantly improved by compensating the apparent loss of stellar flux through modeling of the image formation process within the detector. Multiple observations of a single bright isolated star observed with the IRAC Ch1 instrument are described in Section 2. These observations are analyzed in Section 3 using circular aperture photometry with the recommended 1-dimensional (radial) pixel-phase aperture-flux correction from the IRAC Data Handbook ${ }^{3}$. A new analysis technique called the Lost Flux Method (a.k.a. MATPHOT with residuals) is briefly described in Section 4 and then applied to the observations. A new 2-dimensional pixel-phase aperture-flux flux correction is presented in Section 5 and then applied to the previously measured raw circular aperture fluxes; we demonstrate how the precision of aperture photometry of bright isolated stars corrected with the new 2-dimensional correction can yield photometry that is almost as precise as that produced by the Lost Flux Method. Conclusions are presented in Section 6.

Space Telescopes and Instrumentation 2008: Optical, Infrared, and Millimeter, edited by Jacobus M. Oschmann, Jr., Mattheus W. M. de Graauw, Howard A. MacEwen, Proc. of SPIE Vol. 7010, 70102W, (2008) $\cdot 0277-786$ X/08/\$18 $\cdot$ doi: 10.1117/12.789801 


\section{OBSERVATIONS}

This experiment requires multiple IRAC Ch1 observations with small dither offsets of an isolated bright - but unsaturated - star located in the central region of the IRAC Ch1 field of view. We used the following 16 observations, that were obtained during IRAC Campaign R, because they matched our selection criteria.

Sixteen short (0.4 s) exposure calibration observations of the K0-class star PPM 9412 (a.k.a. HIP 83678, 2MASS J17061029+7340149) were obtained* on 2003 October 8 UT (during Campaign R) with Channel 1 (Ch1) of the Infrared Array Camera (IRAC) instrument onboard the Spitzer Space Telescope after all focus adjustments had been completed. The locations of the star on the array were distributed roughly evenly across a $5 \times 6$ pixel box near the array center.

\section{CIRCULAR APERTURE PHOTOMETRY: PART 1}

\subsection{Raw Flux Measurements}

Circular aperture photometry with a smaller radius of 5 px ( 0.6 arcsec) and a sky annulus spanning 12 to 20 px was done using the IRAF ${ }^{4,5}$ IMEXAMINE task. Figure 1 shows a $4.4 \%$ relative peak-to-peak spread [ $\equiv$ (maximumminimum)/median] in the raw circular aperture flux measurements (FLUX1: open circles). FLUX1 has a median value of 12888 and a semi-interquartile range (SIQR) of 101.5. The relative robust standard deviation $\left[\equiv\left(\mathrm{SIQR} / 0.6745^{\dagger}\right) /\right.$ median] of FLUX1 is $1.2 \%$. To convert FLUX1, and all other flux values in this paper, to an approximate flux density in Janskies, multiply by $3.5 \times 10^{-5}$.

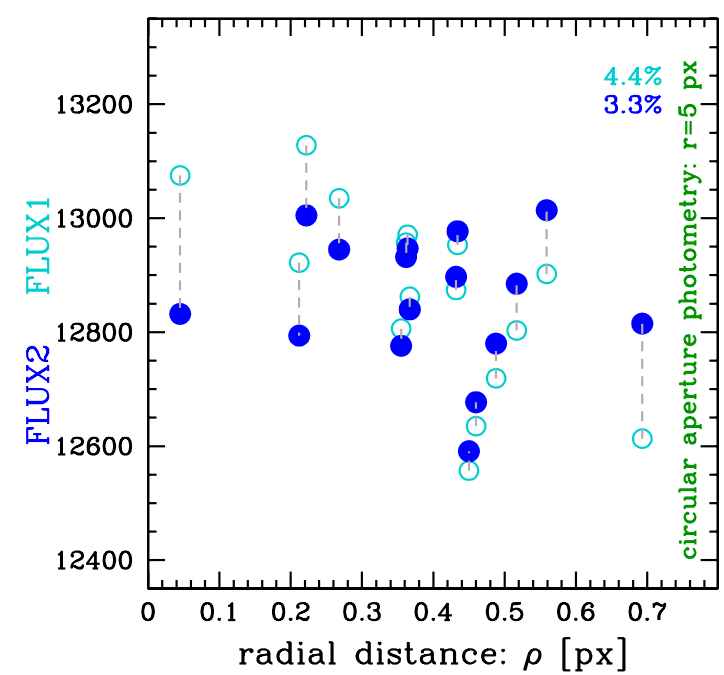

Figure 1. Circular aperture photometry (radius of 5 pixels). The filled (open) circles show the corrected (raw) flux values. The corrected (raw) flux values have a relative robust standard deviation of $0.92 \%(1.2 \%)$.

\footnotetext{
*Observations: ads/sa.spitzer\#00068NNNNN where NNNNN is 75392, 76672, 76928, 77184, 77440, 77696, 77952, $78208,78464,78720,78976,79232,79488,79744,80000,80256$, respectively, for observations 1-16.

${ }^{\dagger}$ The definite integral of a 1-dimensional Gaussian distribution with a mean of zero and a standard deviation of $\sigma$ from minus infinity to $0.6745 \sigma$ is approximately equal to 0.7500 which is the value of the definite integral of a non-normal distribution $f(x)$ with a median value of zero and a semi-interquartile range of SIQR from minus infinity to the value of the SIQR:
}

$$
\int_{-\infty}^{0.6745 \sigma} \frac{1}{\sigma \sqrt{2 \pi}} e^{-\frac{x^{2}}{2 \sigma^{2}}} d x \approx 0.7500 \equiv \int_{-\infty}^{\mathrm{SIQR}} f(x) d x .
$$




\subsection{Flux Correction of the IRAC Data Handbook}

The stellar flux measured from IRAC images depends on exact location where the center of the star falls within the central pixel of the stellar image. This effect, due to the combination of large quantum efficiency variations within individual pixels and the undersampling of the Point Spread Function by the Detector Response Function, is most severe in Channel $1(3.6 \mu \mathrm{m})$ where the correction can be as much as $4 \%$ peak to peak ${ }^{3}$.

For a star with a measured Ch1 flux of $\mathcal{F}$, the IRAC Data Handbook ${ }^{3}$ recommends the following flux correction,

$$
\mathcal{F}^{\prime}=\frac{\mathcal{F}}{\delta_{\rho}},
$$

where the correction factor ${ }^{2,3}$

$$
\delta_{\rho} \equiv 1+0.0535\left[\frac{1}{\sqrt{2 \pi}}-\rho\right]
$$

is a function of the radial distance (pixel phase), in pixel units, from the centroid of the star, located at ( $x, y)$, to the middle of the pixel which contains the centroid,

$$
\rho \equiv \sqrt{\Delta X^{2}+\Delta Y^{2}}
$$

where $\Delta X \equiv(X-0.5)-\operatorname{int}(X-0.5)-0.5$ and $\Delta Y \equiv(Y-0.5)-\operatorname{int}(Y-0.5)-0.5$ are, respectively, the pixelphase offsets in $x$ and $y$ (for the IRAF pixel coordinate system), as shown in Fig. 2. The suggested correction for a pixel-centered star $(\rho=0 \mathrm{px})$ yields a flux reduction of $2.1 \%$ but a corner-centered star $(\rho=1 / \sqrt{2} \approx 0.7071$ px) yields a flux increase of $1.7 \%$. The (flux-weighted) centroid is computed within a 10-pixel radius from the center of the pixel which contains the centroid. The pixel-phase correction in the IRAC Data Handbook was an average derived from stellar images located all over the array, not just in the center, where the data used in this work were taken; since the best focus is near the center of the array, stellar images averaged over the array will be a little broader, and possibly a little more circular, than the stellar images seen in the center.

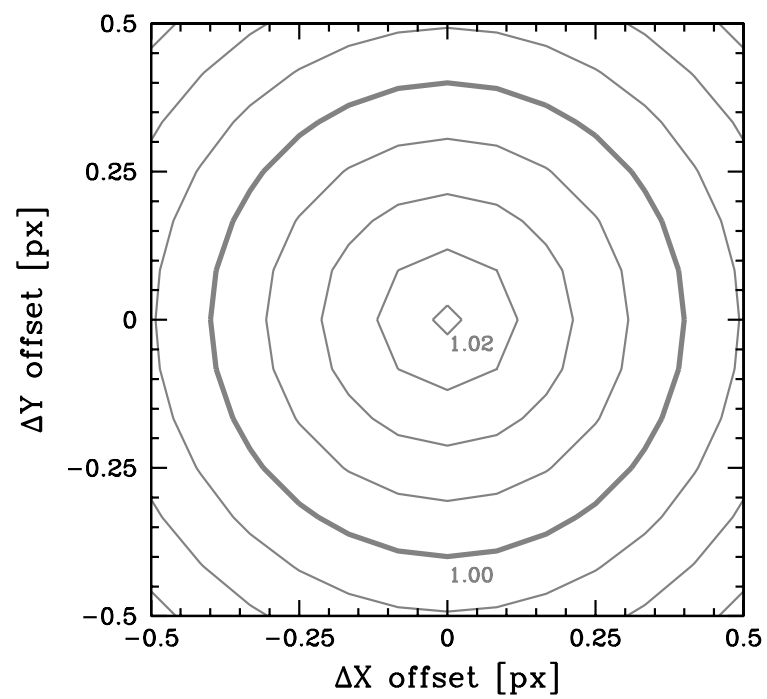

Figure 2. The radial pixel-phase aperture-flux correction (Eq. 2) from the IRAC Data Handbook ${ }^{3}$ as a function of the pixel-phase offset in $x$ and $y$ of the center of the star with respect to the center of the central pixel. The contours range from 1.02 (center) to 0.985 (corners) in steps of 0.005 (102\% to $98.5 \%$ in steps of $0.5 \%$ ).

\subsection{Flux Measurements Corrected Using $\delta_{\rho}$}

Applying the recommended radial Ch1 flux correction $\left(\delta_{\rho}\right.$ : Eq. 2) reduces the relative peak-to-peak spread to $3.3 \%$ (see FLUX2 (filled circles) in Fig. 1). The median and SIQR of FLUX2 is 12862.5 and 79.5, respectively. The relative robust standard deviation of FLUX2 is $0.92 \%$. This is as good as one is likely to do with these observations using the standard calibration procedures recommended by the IRAC Data Handbook. 


\section{MATPHOT (MPDZ) PHOTOMETRY: THE LOST FLUX METHOD}

The MATPHOT algorithm for precise and accurate stellar photometry and astrometry with discrete (sampled) Point Spread Functions (PSFs) has been described in detail ${ }^{6}$. The current ANSI/ISO C language implementation of the MATPHOT algorithm works with user-provided discrete PSFs consisting of a numerical table represented by a matrix in the form of a FITS image. Position partial derivatives are computed ${ }^{7}$ using the following five-point numerical differentiation formula ${ }^{8}$,

$$
f^{\prime}\left(x_{i}\right) \approx \frac{1}{12}\left[f\left(x_{i-2}\right)-8 f\left(x_{i-1}\right)+8 f\left(x_{i+1}\right)-f\left(x_{i+2}\right)\right],
$$

and discrete PSFs are shifted ${ }^{9}$ within an observational model using a 21-pixel-wide damped sinc function,

$$
f^{\text {shifted }}\left(x_{0}\right) \equiv \sum_{i=-10}^{10} f\left(x_{i}\right) \frac{\sin \left(\pi\left(x_{i}-x_{0}\right)\right)}{\pi\left(x_{i}-x_{0}\right)} \exp \left(-\left[\frac{x_{i}-x_{0}}{3.25}\right]^{2}\right),
$$

from the ZODIAC C library written by Marc Buie of Lowell Observatory, which was specifically designed for use with 32-bit floating numbers. Precise and accurate stellar photometry and astrometry are achieved with undersampled CCD observations by using supersampled discrete PSFs that are sampled 2, 3, or more times more finely than the observational data. Although these numerical techniques are not mathematically perfect, they are sufficiently accurate for precision stellar photometry and astrometry due to photon noise which is present in all astronomical imaging observations. The current photometric reduction code ${ }^{\ddagger}$ is based on a robust implementation of the Levenberg-Marquardt method of nonlinear least-squares minimization ${ }^{10-13}$. Detailed analysis of simulated Next Generation Space Telescope observations demonstrate that millipixel relative astrometry and millimag photometric precision should be achievable with complicated space-based discrete $\mathrm{PSFs}^{6}$.

A theoretical $5 \times 5$ supersampled model ${ }^{14}$ of the IRAC PSF in the central region of IRAC Ch1 is shown in Fig. 3. Although the PSF appears to be reasonable with a linear stretch (left) which emphasizes the bright central core, a log stretch (right) shows the numerous weak higher-spatial-frequency features of this very complicated PSF. Hoffmann derived this PSF from CODE V ${ }^{\S}$ ray-tracing models developed at NASA's Goddard Space Flight Center (GSFC). This PSF was computed with a theoretical Rayleigh-Jeans source spectrum incident on the telescope, then integrated over wavelength after taking the product with the detector response vs. wavelength.

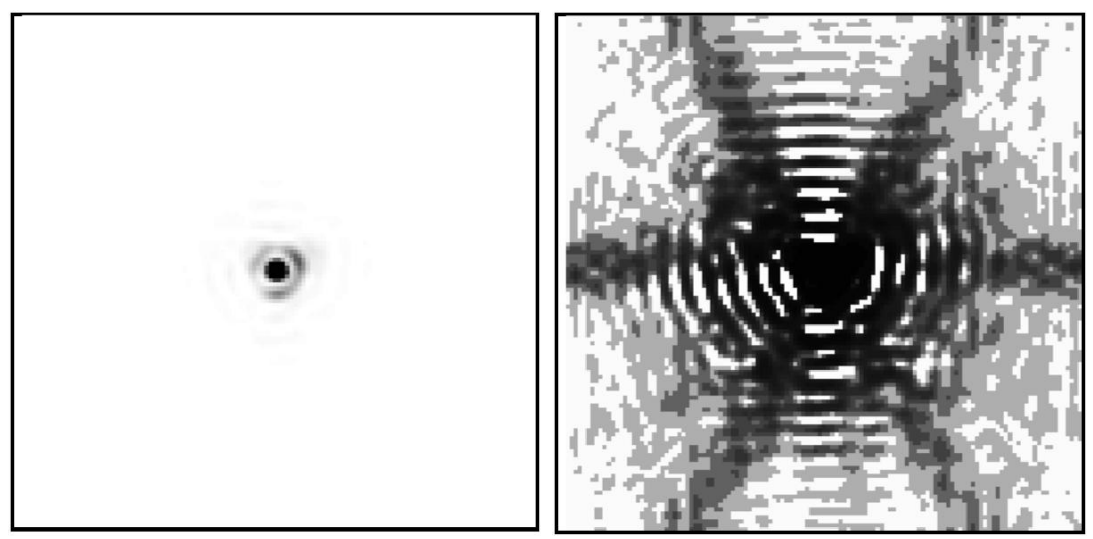

Figure 3. A theoretical $5 \times 5$ supersampled version of the IRAC Ch1 PSF. The left (right) image shows a linear $(\log )$ stretch; black is high and white is low.

\footnotetext{
${ }^{\ddagger}$ All source code and documentation for MATPHOT and support software are freely available at NOAO: http://www.noao.edu/staff/mighell/matphot

$\S$ The CODE V optical design software package is leased from Optical Research Associates, 3280 East Foothill Blvd., Suite 300, Pasadena, CA 91107-3103; http://www.opticalres.com .
} 
The relative intrapixel quantum efficiency variation across a single IRAC Ch1 pixel has been estimated ${ }^{15}$ as follows:

$$
\text { intrapix }=\left(\begin{array}{ccccc}
0.813 & 0.875 & 0.875 & 0.875 & 0.813 \\
0.875 & 1.000 & 1.000 & 1.000 & 0.875 \\
0.875 & 1.000 & 1.000 & 1.000 & 0.875 \\
0.875 & 1.000 & 1.000 & 1.000 & 0.875 \\
0.813 & 0.875 & 0.875 & 0.875 & 0.813
\end{array}\right) .
$$

Each element is the mean RQE (relative quantum efficiency) value, relative to the center of the pixel, over a $0.2 \times 0.2$ pixel $^{2}$ area. Such a variation in QE across a pixel could be obtained if photogenerated charges originating at a pixel edge are more likely to recombine than charges originating near a pixel center, because they must random walk further before being collected. The QE variation is expected to be symmetrical about the center of a pixel, since the InSb layer is opaque over the bandpasses of Channels 1 and 2.

An experimental version of the MATPHOT stellar photometry code, called MPDZ, was developed to simulate and analyze IRAC Ch1 observations ${ }^{16,17}$. MPDZ models the image formation process within IRAC Ch1 by convolving a $5 \times 5$ supersampled PSF with the above $5 \times 5$ relative intrapixel QE variation map for IRAC Ch1.

MATPHOT (MPDZ) photometry was performed on the 16 observations with the theoretical $5 \times 5$ supersampled IRAC Ch1 PSF shown in Fig. 3. The open diamonds in Fig. 4 show a 5.1\% relative peak-to-peak spread in the raw measured stellar flux (FLUX3) values reported by MPDZ. The median and SIQR of FLUX3 is 15831.5 and 121.6, respectively. The relative robust standard deviation of FLUX3 is 1.1\%.
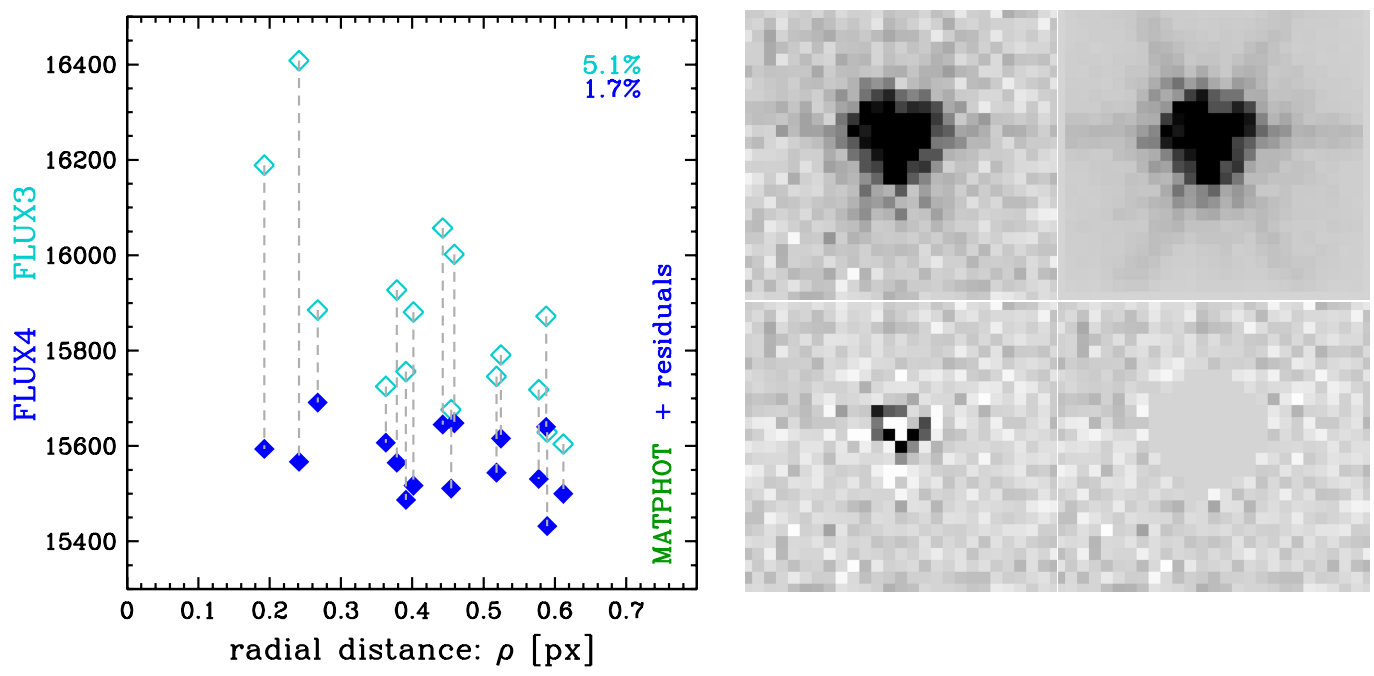

Figure 4. MATPHOT flux measurements.

The upper-left image in Fig. 4 shows the central portion of the first IRAC Ch1 observation. The noiseless best-fit model of the observation is shown in the upper-right image. The residuals remaining after the best-fit model is subtracted from the observation are shown in the lower-left image. The lower-right image is the same as the residual image except that all residuals within a radius of 5 pixels from the fitted center of the star have been set to zero. All of these images are displayed with the same negative linear stretch which was chosen to emphasize the faint features of the stellar image. The filled diamonds in Fig. 4 show a much smaller 1.7\% relative peak-to-peak spread in the FLUX4 values; these flux values are the combination of the raw measured stellar fluxes (open diamonds) with the sum of all the residuals (positive and negative) within a radius of 5 pixels from the fitted center of the star. The median and SIQR of FLUX4 is 15566 and 57, respectively. The relative robust standard deviation of FLUX4 is $0.54 \%$. 
Figure 5 compares the MPDZ photometry with residuals (FLUX4: filled diamonds in Fig. 4) with the corrected circular aperture photometry (FLUX2: filled circles in Fig. 1). The errorbars plotted with the FLUX4 values are the rms errors for the raw MPDZ flux estimates.

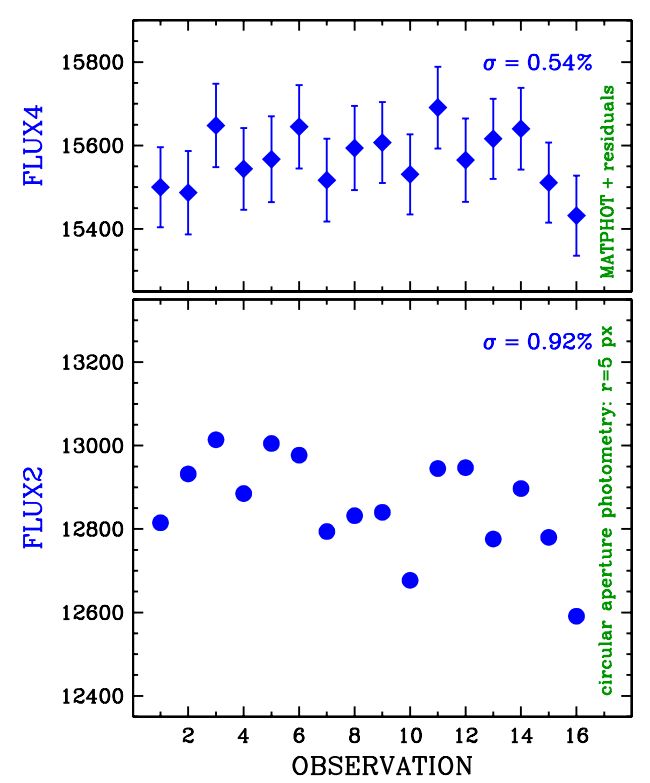

Figure 5. The Lost Flux Method versus circular aperture photometry.

Comparing the Lost Flux Method (a.k.a. MATPHOT with residuals) results with the best results obtained with circular aperture photometry with the recommended radial correction, we see that the precision of photometric measurements have improved significantly using the Lost Flux Method. The relative peak-to-peak spread in independent photometric measurements is a factor of 1.9 smaller (1.7\% vs. 3.3\%); the relative robust standard deviation decreased by a factor of 1.7 (0.92\% vs. 0.54\%).

We see that although the recorded flux of point sources was corrupted by using lossy detectors with large intrapixel quantum efficiency variations, it is possible to significantly improve the precision of stellar photometry from observations made with such detectors - if the image formation process inside the detector is accurately modeled.

Aperture photometry of stellar observations obtained with IRAC Ch1 can be significantly improved by simply dividing the raw measured aperture flux with the MPDZ-computed volume of the Point Response Function (PRF) which is the convolution of the PSF with the discrete Detector Response Function. When the best uncorrected circular aperture flux measurements were divided by the volume of the best-fit PRF computed by MPDZ, the photometric precision improved significantly; the resultant relative peak-to-peak spread is just slightly worse than the spread from the Lost Flux Method results ${ }^{17}$. This suggests that aperture photometry from IRAC Ch1 observations could possibly be significantly improved by using a 2-dimensional correction function rather than the radial (1-dimensional) correction function currently recommended in the IRAC Data Handbook.

\section{CIRCULAR APERTURE PHOTOMETRY: PART 2}

One hundred twenty-one thousand IRAC Ch1 observations of a single star on a flat background were simulated and analyzed with MPDZ. Each stellar observation was simulated using the PSF shown in Fig. 3; a star with an intensity of 63096 electrons was located near the center of an field of $60 \times 60$ pixels on a flat background of 91.01 electrons $\left(\mathrm{e}^{-}\right)\left(B_{\text {true }}=100\right.$ photons $\left.\mathrm{px}^{-1}\right)$ with a readout noise value of $\sigma_{\mathrm{RON}}=3 \mathrm{e}^{-} \mathrm{px}^{-1}$. MPDZ reports the centroid position of the best-fit PRF as well as the intensity-weighted mean centroid of the stellar image - the latter position being the apparent center of the stellar image which generally is not the center of the PSF due to the non-uniform QE response across IRAC Ch1 (Eq. 6). 
The difference between the intensity-weighted mean centroid of the stellar IRAC Ch1 images and the true centroid of the PSF is a systematic error which may be a significant fraction of the undersampled IRAC Ch1 pixel. The cyan (gray) points of the left graph of Fig. 6 show the difference between the intensity-weighted mean centroid of the 121,000 simulated stellar images and the true centroid of the input PSFs $\left(X_{0}, Y_{0}\right)$. The red (black) points show the difference between the centroid of the best-fit PRFs and $\left(X_{0}, Y_{0}\right)$. This graph suggests that the difference between two point sources on any given IRAC Ch1 BCD image may be systematically off by as much as $0.2 \mathrm{px}$ which is 0.24 arcsec on the sky. Figure 7 shows that the intensity-weighted mean centroid errors are separable in $x$ and $y$. Applying the centroid correction functions $\delta_{x}(\Delta X)$ and $\delta_{y}(\Delta Y)$, given in Table 1, to the intensity-weighted mean centroids significantly reduces the centroid errors of the corrected intensity-weighted mean centroids - the centroid errors are now comparable to those estimated by MPDZ (see right graph of Fig. 6).
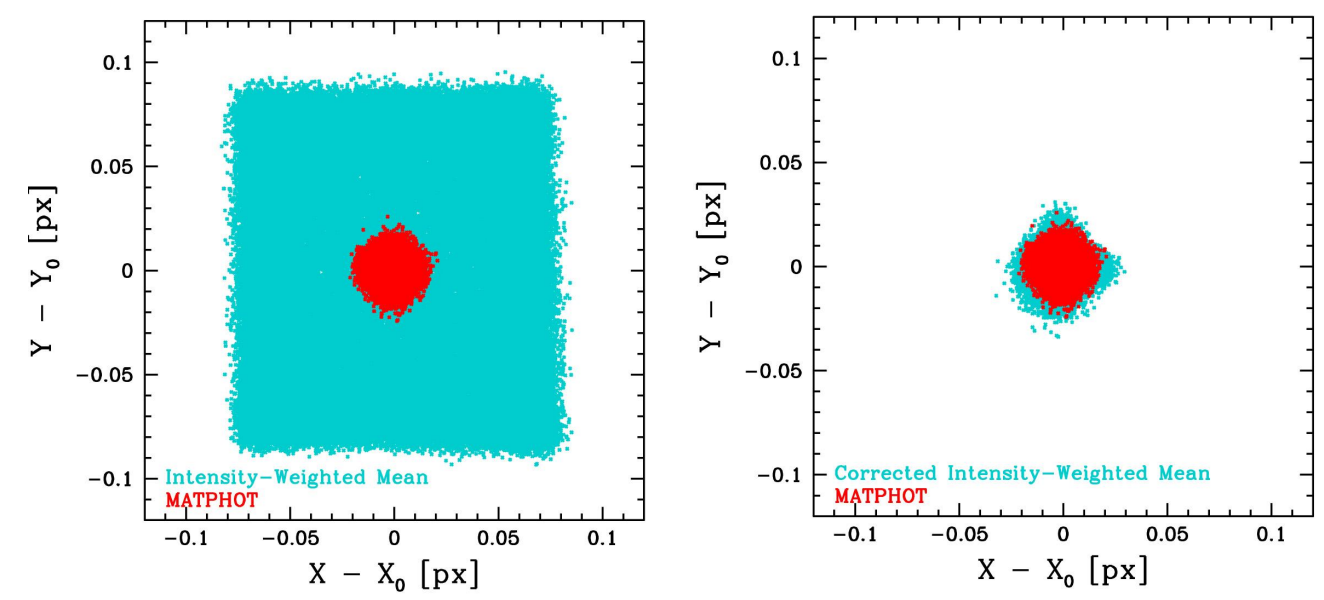

Figure 6. Centroid errors of the 121,000 simulated IRAC Ch1 observations.
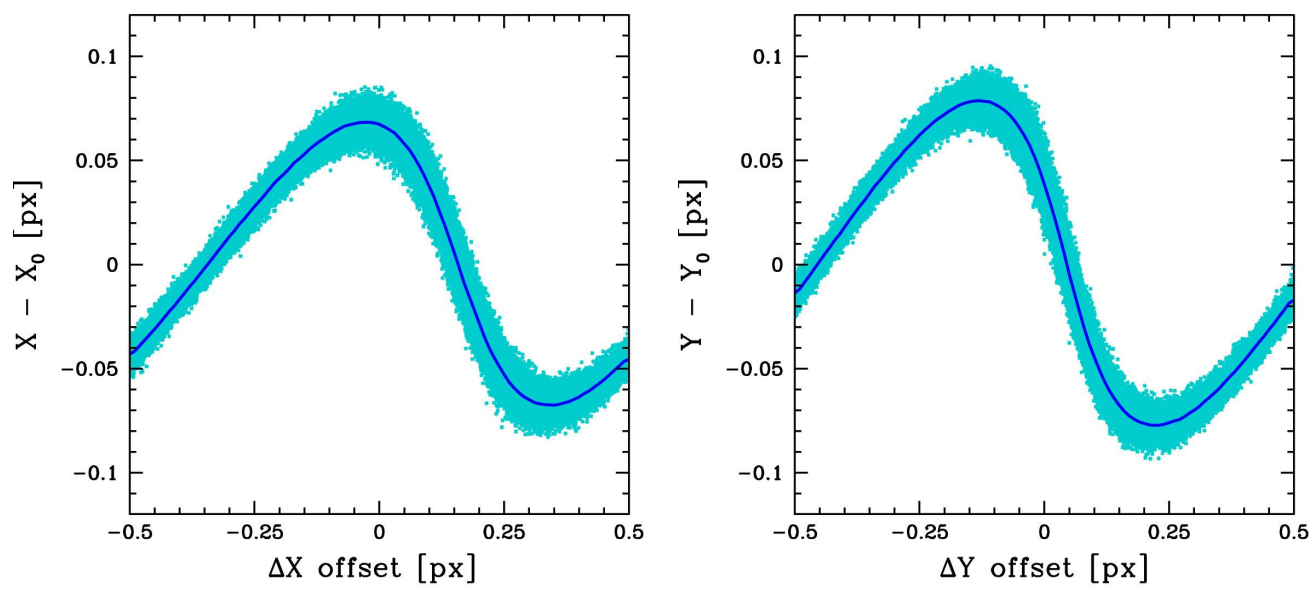

Figure 7. Intensity-weighted mean centroid errors as a function of $\Delta X$ and $\Delta Y$ pixel-phase offsets. The blue curve on the left [right] graph is the centroid correction function $\delta_{x}(\Delta X)\left[\delta_{y}(\Delta Y)\right]$ given in Table 1. 
Table 1. Centroid Corrections

\begin{tabular}{|c|c|c|c|c|c|}
\hline$\Delta$ & $\delta_{x}$ & $\delta_{y}$ & $\Delta$ & $\delta_{x}$ & $\delta_{y}$ \\
\hline-0.50 & -0.0429 & -0.0136 & 0.00 & 0.0674 & 0.0388 \\
\hline-0.49 & -0.0416 & -0.0118 & 0.01 & 0.0662 & 0.0313 \\
\hline-0.48 & -0.0390 & -0.0085 & 0.02 & 0.0648 & 0.0230 \\
\hline-0.47 & -0.0363 & -0.0053 & 0.03 & 0.0633 & 0.0143 \\
\hline-0.46 & -0.0337 & -0.0019 & 0.04 & 0.0608 & 0.0053 \\
\hline-0.45 & -0.0309 & 0.0017 & 0.05 & 0.0581 & -0.0045 \\
\hline-0.44 & -0.0282 & 0.0049 & 0.06 & 0.0552 & -0.0136 \\
\hline-0.43 & -0.0253 & 0.0081 & 0.07 & 0.0515 & -0.0223 \\
\hline-0.42 & -0.0224 & 0.0115 & 0.08 & 0.0474 & -0.0305 \\
\hline-0.41 & -0.0196 & 0.0146 & 0.09 & 0.0428 & -0.0381 \\
\hline-0.40 & -0.0166 & 0.0180 & 0.10 & 0.0374 & -0.0445 \\
\hline-0.39 & -0.0137 & 0.0214 & 0.11 & 0.0322 & -0.0504 \\
\hline-0.38 & -0.0107 & 0.0247 & 0.12 & 0.0258 & -0.0558 \\
\hline-0.37 & -0.0078 & 0.0280 & 0.13 & 0.0197 & -0.0604 \\
\hline-0.36 & -0.0048 & 0.0309 & 0.14 & 0.0126 & -0.0643 \\
\hline-0.35 & -0.0017 & 0.0341 & 0.15 & 0.0058 & -0.0676 \\
\hline-0.34 & 0.0012 & 0.0376 & 0.16 & -0.0015 & -0.0704 \\
\hline-0.33 & 0.0044 & 0.0404 & 0.17 & -0.0088 & -0.0726 \\
\hline-0.32 & 0.0075 & 0.0432 & 0.18 & -0.0155 & -0.0743 \\
\hline-0.31 & 0.0105 & 0.0462 & 0.19 & -0.0217 & -0.0755 \\
\hline-0.30 & 0.0135 & 0.0491 & 0.20 & -0.0281 & -0.0763 \\
\hline-0.29 & 0.0164 & 0.0518 & 0.21 & -0.0344 & -0.0770 \\
\hline-0.28 & 0.0195 & 0.0548 & 0.22 & -0.0399 & -0.0773 \\
\hline-0.27 & 0.0221 & 0.0572 & 0.23 & -0.0448 & -0.0771 \\
\hline-0.26 & 0.0250 & 0.0596 & 0.24 & -0.0488 & -0.0767 \\
\hline-0.25 & 0.0279 & 0.0622 & 0.25 & -0.0528 & -0.0759 \\
\hline-0.24 & 0.0306 & 0.0646 & 0.26 & -0.0564 & -0.0752 \\
\hline-0.23 & 0.0334 & 0.0666 & 0.27 & -0.0593 & -0.0745 \\
\hline-0.22 & 0.0362 & 0.0687 & 0.28 & -0.0616 & -0.0731 \\
\hline-0.21 & 0.0391 & 0.0705 & 0.29 & -0.0635 & -0.0715 \\
\hline-0.20 & 0.0416 & 0.0722 & 0.30 & -0.0649 & -0.0700 \\
\hline-0.19 & 0.0438 & 0.0739 & 0.31 & -0.0662 & -0.0681 \\
\hline-0.18 & 0.0463 & 0.0752 & 0.32 & -0.0669 & -0.0659 \\
\hline-0.17 & 0.0490 & 0.0766 & 0.33 & -0.0673 & -0.0640 \\
\hline-0.16 & 0.0510 & 0.0775 & 0.34 & -0.0674 & -0.0619 \\
\hline-0.15 & 0.0531 & 0.0782 & 0.35 & -0.0675 & -0.0595 \\
\hline-0.14 & 0.0554 & 0.0786 & 0.36 & -0.0671 & -0.0569 \\
\hline-0.13 & 0.0573 & 0.0787 & 0.37 & -0.0665 & -0.0542 \\
\hline-0.12 & 0.0591 & 0.0785 & 0.38 & -0.0658 & -0.0519 \\
\hline-0.11 & 0.0607 & 0.0782 & 0.39 & -0.0648 & -0.0491 \\
\hline-0.10 & 0.0624 & 0.0772 & 0.40 & -0.0636 & -0.0462 \\
\hline-0.09 & 0.0639 & 0.0759 & 0.41 & -0.0620 & -0.0433 \\
\hline-0.08 & 0.0652 & 0.0742 & 0.42 & -0.0607 & -0.0403 \\
\hline-0.07 & 0.0665 & 0.0720 & 0.43 & -0.0590 & -0.0372 \\
\hline-0.06 & 0.0672 & 0.0693 & 0.44 & -0.0571 & -0.0344 \\
\hline-0.05 & 0.0677 & 0.0659 & 0.45 & -0.0553 & -0.0312 \\
\hline-0.04 & 0.0682 & 0.0620 & 0.46 & -0.0532 & -0.0283 \\
\hline-0.03 & 0.0684 & 0.0574 & 0.47 & -0.0510 & -0.0250 \\
\hline-0.02 & 0.0683 & 0.0520 & 0.48 & -0.0487 & -0.0219 \\
\hline-0.01 & 0.0681 & 0.0458 & 0.49 & -0.0465 & -0.0185 \\
\hline
\end{tabular}

A new 2-dimensional pixel-phase aperture-flux correction was derived in the following manner. We established a grid of $13 \times 13$ points spanning the range of values for $\Delta X$ and $\Delta Y$ pixel-phase offsets (-0.5 to 0.5$)$ with steps of $1 / 12^{\text {th }}$ of a pixel. The median PRF volume, reported by MPDZ, of the 121,000 simulated IRAC Ch1 observations found within $1 / 24^{\text {th }}$ of a pixel in $x$ and $y$ was determined for each grid point and was then calibrated in the same manner as the recommended radial correction (Eq. 2) by dividing by the value of the median PRF volume of the all of the 121,000 simulated observations (0.908189). The resulting new 2-dimensional pixel-phase aperture-flux correction function, $\delta_{x y}(\Delta X, \Delta Y)$, is given in Table 2 and is shown graphically in Fig. 8. 
Table 2. New 2-d Pixel-Phase Aperture-Flux Correction Function: $\delta_{x y}(\Delta X, \Delta Y)$

\begin{tabular}{|c|c|c|c|c|c|c|c|c|c|}
\hline$\overline{\Delta X X}$ & $\overline{\Delta Y}$ & $\rho$ & $\overline{\overline{\delta_{\rho}}}$ & $\overline{\delta_{x y}}$ & $\overline{\Delta X}$ & $\overline{\Delta \Delta Y}$ & $\overline{\rho \rho}$ & $\overline{\overline{\delta_{\rho}}}$ & $\overline{\delta_{x y}}$ \\
\hline-0.5000 & -0.5000 & 0.7071 & 0.9835 & 0.9819 & 0.0833 & 0.0000 & 0.0833 & 1.0169 & 1.0205 \\
\hline-0.4167 & -0.5000 & 0.6509 & 0.9865 & 0.9797 & 0.1667 & 0.0000 & 0.1667 & 1.0124 & 1.0215 \\
\hline-0.3333 & -0.5000 & 0.6009 & 0.9892 & 0.9788 & 0.2500 & 0.0000 & 0.2500 & 1.0080 & 1.0198 \\
\hline-0.2500 & -0.5000 & 0.5590 & 0.9914 & 0.9806 & 0.3333 & 0.0000 & 0.3333 & 1.0035 & 1.0156 \\
\hline-0.1667 & -0.5000 & 0.5270 & 0.9931 & 0.9850 & 0.4167 & 0.0000 & 0.4167 & 0.9991 & 1.0102 \\
\hline-0.0833 & -0.5000 & 0.5069 & 0.9942 & 0.9897 & 0.5000 & 0.0000 & 0.5000 & 0.9946 & 1.0061 \\
\hline 0.0000 & -0.5000 & 0.5000 & 0.9946 & 0.9945 & -0.5000 & 0.0833 & 0.5069 & 0.9942 & 1.0036 \\
\hline 0.0833 & -0.5000 & 0.5069 & 0.9942 & 0.9979 & -0.4167 & 0.0833 & 0.4249 & 0.9986 & 1.0013 \\
\hline 0.1667 & -0.5000 & 0.5270 & 0.9931 & 0.9988 & -0.3333 & 0.0833 & 0.3436 & 1.0030 & 1.0006 \\
\hline 0.2500 & -0.5000 & 0.5590 & 0.9914 & 0.9973 & -0.2500 & 0.0833 & 0.2635 & 1.0072 & 1.0024 \\
\hline 0.3333 & -0.5000 & 0.6009 & 0.9892 & 0.9933 & -0.1667 & 0.0833 & 0.1863 & 1.0114 & 1.0064 \\
\hline 0.4167 & -0.5000 & 0.6509 & 0.9865 & 0.9879 & -0.0833 & 0.0833 & 0.1179 & 1.0150 & 1.0116 \\
\hline 0.5000 & -0.5000 & 0.7071 & 0.9835 & 0.9841 & 0.0000 & 0.0833 & 0.0833 & 1.0169 & 1.0168 \\
\hline-0.5000 & -0.4167 & 0.6509 & 0.9865 & 0.9826 & 0.0833 & 0.0833 & 0.1179 & 1.0150 & 1.0200 \\
\hline-0.4167 & -0.4167 & 0.5893 & 0.9898 & 0.9804 & 0.1667 & 0.0833 & 0.1863 & 1.0114 & 1.0212 \\
\hline-0.3333 & -0.4167 & 0.5336 & 0.9928 & 0.9796 & 0.2500 & 0.0833 & 0.2635 & 1.0072 & 1.0194 \\
\hline-0.2500 & -0.4167 & 0.4859 & 0.9953 & 0.9815 & 0.3333 & 0.0833 & 0.3436 & 1.0030 & 1.0153 \\
\hline-0.1667 & -0.4167 & 0.4488 & 0.9973 & 0.9857 & 0.4167 & 0.0833 & 0.4249 & 0.9986 & 1.0097 \\
\hline-0.0833 & -0.4167 & 0.4249 & 0.9986 & 0.9907 & 0.5000 & 0.0833 & 0.5069 & 0.9942 & 1.0058 \\
\hline 0.0000 & -0.4167 & 0.4167 & 0.9991 & 0.9953 & -0.5000 & 0.1667 & 0.5270 & 0.9931 & 1.0005 \\
\hline 0.0833 & -0.4167 & 0.4249 & 0.9986 & 0.9987 & -0.4167 & 0.1667 & 0.4488 & 0.9973 & 0.9982 \\
\hline 0.1667 & -0.4167 & 0.4488 & 0.9973 & 0.9996 & -0.3333 & 0.1667 & 0.3727 & 1.0014 & 0.9974 \\
\hline 0.2500 & -0.4167 & 0.4859 & 0.9953 & 0.9980 & -0.2500 & 0.1667 & 0.3005 & 1.0053 & 0.9993 \\
\hline 0.3333 & -0.4167 & 0.5336 & 0.9928 & 0.9941 & -0.1667 & 0.1667 & 0.2357 & 1.0087 & 1.0033 \\
\hline 0.4167 & -0.4167 & 0.5893 & 0.9898 & 0.9887 & -0.0833 & 0.1667 & 0.1863 & 1.0114 & 1.0084 \\
\hline 0.5000 & -0.4167 & 0.6509 & 0.9865 & 0.9850 & 0.0000 & 0.1667 & 0.1667 & 1.0124 & 1.0133 \\
\hline-0.5000 & -0.3333 & 0.6009 & 0.9892 & 0.9859 & 0.0833 & 0.1667 & 0.1863 & 1.0114 & 1.0168 \\
\hline-0.4167 & -0.3333 & 0.5336 & 0.9928 & 0.9836 & 0.1667 & 0.1667 & 0.2357 & 1.0087 & 1.0178 \\
\hline-0.3333 & -0.3333 & 0.4714 & 0.9961 & 0.9830 & 0.2500 & 0.1667 & 0.3005 & 1.0053 & 1.0160 \\
\hline-0.2500 & -0.3333 & 0.4167 & 0.9991 & 0.9847 & 0.3333 & 0.1667 & 0.3727 & 1.0014 & 1.0121 \\
\hline-0.1667 & -0.3333 & 0.3727 & 1.0014 & 0.9888 & 0.4167 & 0.1667 & 0.4488 & 0.9973 & 1.0066 \\
\hline-0.0833 & -0.3333 & 0.3436 & 1.0030 & 0.9940 & 0.5000 & 0.1667 & 0.5270 & 0.9931 & 1.0026 \\
\hline 0.0000 & -0.3333 & 0.3333 & 1.0035 & 0.9989 & -0.5000 & 0.2500 & 0.5590 & 0.9914 & 0.9953 \\
\hline 0.0833 & -0.3333 & 0.3436 & 1.0030 & 1.0022 & -0.4167 & 0.2500 & 0.4859 & 0.9953 & 0.9929 \\
\hline 0.1667 & -0.3333 & 0.3727 & 1.0014 & 1.0030 & -0.3333 & 0.2500 & 0.4167 & 0.9991 & 0.9920 \\
\hline 0.2500 & -0.3333 & 0.4167 & 0.9991 & 1.0012 & -0.2500 & 0.2500 & 0.3536 & 1.0024 & 0.9938 \\
\hline 0.3333 & -0.3333 & 0.4714 & 0.9961 & 0.9975 & -0.1667 & 0.2500 & 0.3005 & 1.0053 & 0.9981 \\
\hline 0.4167 & -0.3333 & 0.5336 & 0.9928 & 0.9921 & -0.0833 & 0.2500 & 0.2635 & 1.0072 & 1.0033 \\
\hline 0.5000 & -0.3333 & 0.6009 & 0.9892 & 0.9880 & 0.0000 & 0.2500 & 0.2500 & 1.0080 & 1.0080 \\
\hline-0.5000 & -0.2500 & 0.5590 & 0.9914 & 0.9911 & 0.0833 & 0.2500 & 0.2635 & 1.0072 & 1.0115 \\
\hline-0.4167 & -0.2500 & 0.4859 & 0.9953 & 0.9888 & 0.1667 & 0.2500 & 0.3005 & 1.0053 & 1.0123 \\
\hline-0.3333 & -0.2500 & 0.4167 & 0.9991 & 0.9879 & 0.2500 & 0.2500 & 0.3536 & 1.0024 & 1.0108 \\
\hline-0.2500 & -0.2500 & 0.3536 & 1.0024 & 0.9898 & 0.3333 & 0.2500 & 0.4167 & 0.9991 & 1.0066 \\
\hline-0.1667 & -0.2500 & 0.3005 & 1.0053 & 0.9939 & 0.4167 & 0.2500 & 0.4859 & 0.9953 & 1.0014 \\
\hline-0.0833 & -0.2500 & 0.2635 & 1.0072 & 0.9991 & 0.5000 & 0.2500 & 0.5590 & 0.9914 & 0.9974 \\
\hline 0.0000 & -0.2500 & 0.2500 & 1.0080 & 1.0041 & -0.5000 & 0.3333 & 0.6009 & 0.9892 & 0.9894 \\
\hline 0.0833 & -0.2500 & 0.2635 & 1.0072 & 1.0074 & -0.4167 & 0.3333 & 0.5336 & 0.9928 & 0.9872 \\
\hline 0.1667 & -0.2500 & 0.3005 & 1.0053 & 1.0084 & -0.3333 & 0.3333 & 0.4714 & 0.9961 & 0.9860 \\
\hline 0.2500 & -0.2500 & 0.3536 & 1.0024 & 1.0066 & -0.2500 & 0.3333 & 0.4167 & 0.9991 & 0.9882 \\
\hline 0.3333 & -0.2500 & 0.4167 & 0.9991 & 1.0026 & -0.1667 & 0.3333 & 0.3727 & 1.0014 & 0.9922 \\
\hline 0.4167 & -0.2500 & 0.4859 & 0.9953 & 0.9973 & -0.0833 & 0.3333 & 0.3436 & 1.0030 & 0.9973 \\
\hline 0.5000 & -0.2500 & 0.5590 & 0.9914 & 0.9933 & 0.0000 & 0.3333 & 0.3333 & 1.0035 & 1.0018 \\
\hline-0.5000 & -0.1667 & 0.5270 & 0.9931 & 0.9968 & 0.0833 & 0.3333 & 0.3436 & 1.0030 & 1.0053 \\
\hline-0.4167 & -0.1667 & 0.4488 & 0.9973 & 0.9946 & 0.1667 & 0.3333 & 0.3727 & 1.0014 & 1.0061 \\
\hline-0.3333 & -0.1667 & 0.3727 & 1.0014 & 0.9936 & 0.2500 & 0.3333 & 0.4167 & 0.9991 & 1.0046 \\
\hline-0.2500 & -0.1667 & 0.3005 & 1.0053 & 0.9957 & 0.3333 & 0.3333 & 0.4714 & 0.9961 & 1.0008 \\
\hline-0.1667 & -0.1667 & 0.2357 & 1.0087 & 0.9998 & 0.4167 & 0.3333 & 0.5336 & 0.9928 & 0.9953 \\
\hline-0.0833 & -0.1667 & 0.1863 & 1.0114 & 1.0051 & 0.5000 & 0.3333 & 0.6009 & 0.9892 & 0.9915 \\
\hline 0.0000 & -0.1667 & 0.1667 & 1.0124 & 1.0100 & -0.5000 & 0.4167 & 0.6509 & 0.9865 & 0.9842 \\
\hline 0.0833 & -0.1667 & 0.1863 & 1.0114 & 1.0134 & -0.4167 & 0.4167 & 0.5893 & 0.9898 & 0.9820 \\
\hline 0.1667 & -0.1667 & 0.2357 & 1.0087 & 1.0142 & -0.3333 & 0.4167 & 0.5336 & 0.9928 & 0.9813 \\
\hline 0.2500 & -0.1667 & 0.3005 & 1.0053 & 1.0124 & -0.2500 & 0.4167 & 0.4859 & 0.9953 & 0.9832 \\
\hline 0.3333 & -0.1667 & 0.3727 & 1.0014 & 1.0084 & -0.1667 & 0.4167 & 0.4488 & 0.9973 & 0.9872 \\
\hline 0.4167 & -0.1667 & 0.4488 & 0.9973 & 1.0032 & -0.0833 & 0.4167 & 0.4249 & 0.9986 & 0.9922 \\
\hline 0.5000 & -0.1667 & 0.5270 & 0.9931 & 0.9989 & 0.0000 & 0.4167 & 0.4167 & 0.9991 & 0.9971 \\
\hline-0.5000 & -0.0833 & 0.5069 & 0.9942 & 1.0013 & 0.0833 & 0.4167 & 0.4249 & 0.9986 & 1.0003 \\
\hline-0.4167 & -0.0833 & 0.4249 & 0.9986 & 0.9993 & 0.1667 & 0.4167 & 0.4488 & 0.9973 & 1.0011 \\
\hline-0.3333 & -0.0833 & 0.3436 & 1.0030 & 0.9985 & 0.2500 & 0.4167 & 0.4859 & 0.9953 & 0.9997 \\
\hline-0.2500 & -0.0833 & 0.2635 & 1.0072 & 1.0003 & 0.3333 & 0.4167 & 0.5336 & 0.9928 & 0.9958 \\
\hline-0.1667 & -0.0833 & 0.1863 & 1.0114 & 1.0044 & 0.4167 & 0.4167 & 0.5893 & 0.9898 & 0.9905 \\
\hline-0.0833 & -0.0833 & 0.1179 & 1.0150 & 1.0098 & 0.5000 & 0.4167 & 0.6509 & 0.9865 & 0.9864 \\
\hline 0.0000 & -0.0833 & 0.0833 & 1.0169 & 1.0146 & -0.5000 & 0.5000 & 0.7071 & 0.9835 & 0.9820 \\
\hline 0.0833 & -0.0833 & 0.1179 & 1.0150 & 1.0180 & -0.4167 & 0.5000 & 0.6509 & 0.9865 & 0.9798 \\
\hline 0.1667 & -0.0833 & 0.1863 & 1.0114 & 1.0191 & -0.3333 & 0.5000 & 0.6009 & 0.9892 & 0.9791 \\
\hline 0.2500 & -0.0833 & 0.2635 & 1.0072 & 1.0174 & -0.2500 & 0.5000 & 0.5590 & 0.9914 & 0.9807 \\
\hline 0.3333 & -0.0833 & 0.3436 & 1.0030 & 1.0133 & -0.1667 & 0.5000 & 0.5270 & 0.9931 & 0.9846 \\
\hline 0.4167 & -0.0833 & 0.4249 & 0.9986 & 1.0078 & -0.0833 & 0.5000 & 0.5069 & 0.9942 & 0.9900 \\
\hline 0.5000 & -0.0833 & 0.5069 & 0.9942 & 1.0036 & 0.0000 & 0.5000 & 0.5000 & 0.9946 & 0.9945 \\
\hline-0.5000 & 0.0000 & 0.5000 & 0.9946 & 1.0039 & 0.0833 & 0.5000 & 0.5069 & 0.9942 & 0.9980 \\
\hline-0.4167 & 0.0000 & 0.4167 & 0.9991 & 1.0017 & 0.1667 & 0.5000 & 0.5270 & 0.9931 & 0.9989 \\
\hline-0.3333 & 0.0000 & 0.3333 & 1.0035 & 1.0009 & 0.2500 & 0.5000 & 0.5590 & 0.9914 & 0.9974 \\
\hline-0.2500 & 0.0000 & 0.2500 & 1.0080 & 1.0027 & 0.3333 & 0.5000 & 0.6009 & 0.9892 & 0.9934 \\
\hline-0.1667 & 0.0000 & 0.1667 & 1.0124 & 1.0069 & 0.4167 & 0.5000 & 0.6509 & 0.9865 & 0.9883 \\
\hline-0.0833 & 0.0000 & 0.0833 & 1.0169 & 1.0122 & 0.5000 & 0.5000 & 0.7071 & 0.9835 & 0.9843 \\
\hline 0.0000 & 0.0000 & 0.0000 & 1.0213 & 1.0172 & & & & & \\
\hline
\end{tabular}

Proc. of SPIE Vol. $701070102 \mathrm{~W}-9$ 

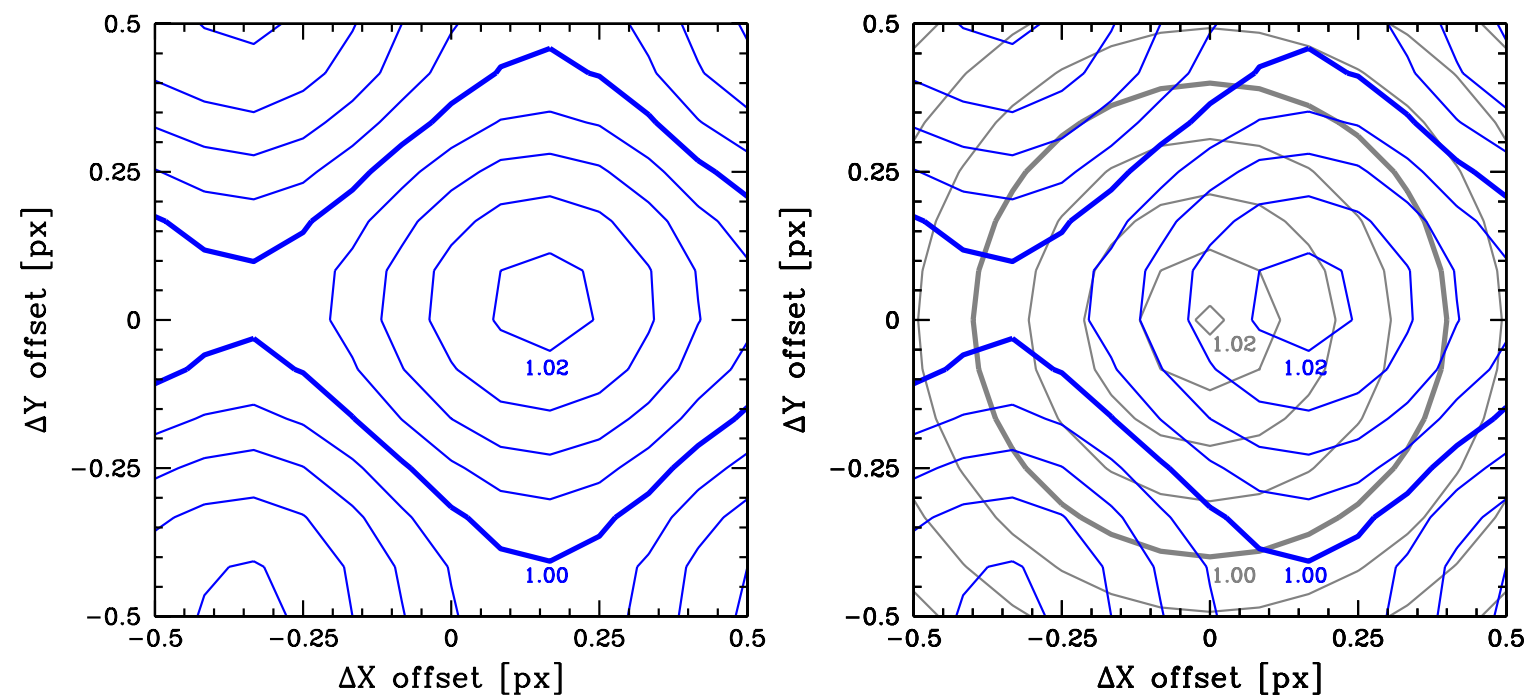

Figure 8. (left) The new 2-dimensional pixel-phase aperture-flux correction (Table 2) as a function of the pixelphase offset in $x$ and $y$ of the center of the star with respect to the center of the central pixel. The contours range from 1.02 (center) to 0.98 (left corners) in steps of 0.005 (102\% to $98 \%$ in steps of $0.5 \%$ ). (right) The new 2dimensional correction (blue/black contours) compared with the recommended radial correction (gray contours).

Correcting the FLUX1 circular aperture fluxes (from Section 3) with the new 2-dimensional pixel-phase aperture-flux correction function, $\delta_{x y}(\Delta X, \Delta Y)$, yields a significant gain in photometric precision over that obtained with the recommended radial correction: the robust relative standard deviation improved by a factor of 1.48 (from $0.92 \%$ to $0.62 \%$ : see Fig. 8 ). With the new correction, one can now achieve photometric precision with aperture photometry on bright isolated stars that is comparable to the best results produced by PSF-fitting photometric procedures $(0.62 \%$ versus $0.54 \%)$ !

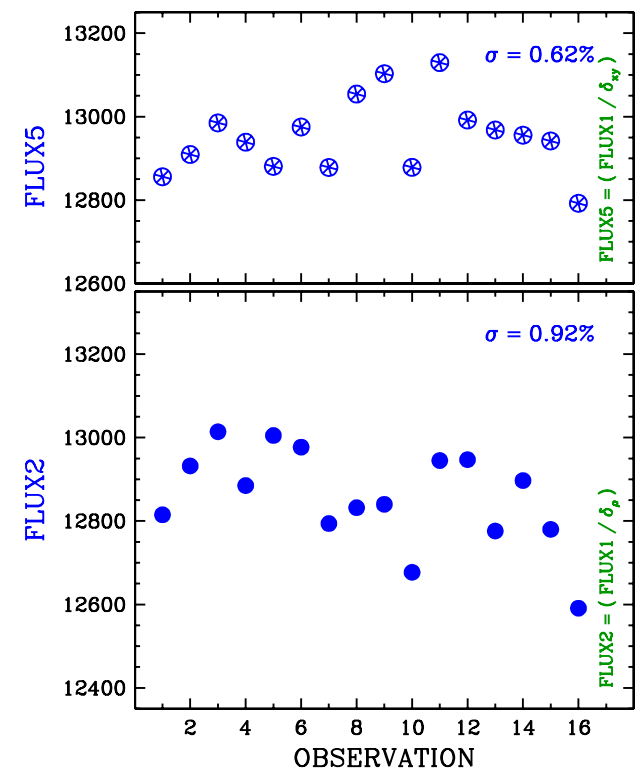

Figure 9. Photometric precision with circular aperture photometry corrected with the new 2-dimensional pixelphase aperture-flux correction (top) versus the recommended radial correction (bottom). 


\section{CONCLUSION}

Planning is underway for a possible post-cryogenic mission with the Spitzer Space Telescope. Only Channels 1 and $2(3.6$ and $4.5 \mu \mathrm{m})$ of the Infrared Array Camera will be operational at that time; they will have unmatched sensitivity from 3 to 5 microns until the James Webb Space Telescope is launched. The new 2-dimensional pixelphase aperture-flux correction should enhance the science return not only of existing IRAC Ch1 observations in the Spitzer Data Archive but also those that might be made during Spitzer's possible Warm Mission phase which would start around April 2009 after all of the cryogen is depleted.

Current near-infrared detector technology can produce space-based astronomical imagers with non-uniform pixel response functions. Large intrapixel quantum efficiency variations can cause significant loss of stellar flux depending on where a star is centered within the central pixel of an undersampled stellar image. This article showed how the precision of aperture stellar photometry from an existing space-based near-infrared camera with a lossy detector can be significantly improved by compensating the apparent loss of stellar flux by accurately modeling the image formation process within the detector.

\section{ACKNOWLEDGMENTS}

We wish to thank David Elliott, Patrick Lowrance, and the rest of the IRAC Instrument Team for their support of this research effort. I also wish to thank Mike Merrill and Ron Probst for many useful discussions about state-of-the-art near-infrared detectors. This work has been supported by a grant from the National Aeronautics and Space Administration (NASA), Interagency Order No. NNG06EC81I which was awarded by the Applied Information Systems Research (AISR) Program of NASA's Science Mission Directorate. Additional support was provided by an award issued by JPL/Caltech (Spitzer Space Telescope Cycle 4 Archive Proposal \# 40106; Subcontract No. 1311641).

\section{REFERENCES}

[1] Fazio G. G., et al., "The Infrared Array Camera (IRAC) for the Spitzer Space Telescope," ApJS 154, 10-17 (2004).

[2] Reach, W. T., et al., "Absolute Calibration of the Infrared Array Camera on the Spitzer Space Telescope," PASP 117, 978-990 (2005).

[3] Reach, W. T., et al., [Infrared Array Camera Data Handbook, Version 3.0 (January 20, 2006)], Spitzer Science Center, Pasadena (2006).

[4] Tody, D., "The IRAF Data Reduction and Analysis System," Proc. SPIE 627, 733+ (1986).

[5] Tody, D., "IRAF in the Nineties," ASP Conference Series 52: Astronomical Data Analysis Software and Systems II , 173-183 (1993).

[6] Mighell, K. J., "Stellar photometry and astrometry with discrete point spread functions," MNRAS 362, $861-878$ (2005).

[7] Mighell, K. J., "The MATPHOT Algorithm for Digital Point Spread Function CCD Stellar Photometry," ASP Conference Series 281: Astronomical Data Analysis Software and Systems XI, 387-391 (2002).

[8] Abramowitz, M. and Stegun, I., [Handbook of Mathematical Functions with Formulas, Graphs, and Mathematical Tables], Applied Mathematics Series, 55, eds. M. Abromowitz and I. Stegun, NBS, Washington, D.C. (1964).

[9] Mighell, K. J., "The MATPHOT algorithm for digital point spread function CCD stellar photometry," Proc. SPIE 4847, 207-216 (2002).

[10] Levenberg, K., "A method for the solution of certain problems in least squares," Quarterly of Applied Mathematics 2, 164-168 (1944).

[11] Marquardt, D., "An algorithm for least-squares estimation of nonlinear parameters," SIAM Journal of Applied Mathematics 11, 431-441 (1963).

[12] Mighell, K. J., "Accurate stellar photometry in crowded fields," MNRAS 238, 807-833 (1989).

[13] Mighell, K. J., "Algorithms for CCD stellar photometry," ASP Conference Series 172: Astronomical Data Analysis Software and Systems VIII, 317-328 (1999). 
[14] Hoffmann, B., "25 Position Model Pixel Response Functions (PRF) Description and Quality," IRAC/TM05014 (Simfit Report 52 Final) (2005).

[15] Hoffmann, B., "Intra-pixel Variation Effect on Aperture Photometry," IRAC/TM05-028 (Simfit Report 59; Version 2: December 10, 2005) (2005).

[16] Mighell, K. J., "Innovative image analysis software as a technology driver for advances in space telescope design," Proc. SPIE 6265, 6265T (2006).

[17] Mighell, K. J., "The Lost Flux Method: A New Algorithm for Improving the Precision of Space-Based Near-Infrared Stellar Photometry with Lossy Detectors," ASP Conference Series 281: Astronomical Data Analysis Software and Systems XI, 405 (2007). 